\title{
MEASUREMENT AND SIMULATION OF A 17 GHz PHOTONIC BAND GAP STRUCTURE FOR ACCELERATOR APPLICATIONS
}

\author{
M.A.Shapiro, W.J.Brown, C.Chen, J.R.Sirigiri, E.I.Smirnova, and R.J.Temkin \\ Plasma Science and Fusion Center, MIT, Cambridge, MA 02139, USA
}

\begin{abstract}
A $17 \mathrm{GHz}$ photonic band gap (PBG) cavity for a linear accelerator is designed and experimentally studied in the cold test. The cavity is built of a triangular lattice of metal rods with a defect in the center. The cold test results are in good agreement with the theory. The longitudinal and transverse wake potentials in the PBG cavity are calculated and found to be smaller than those in a conventional (pillbox) structure.
\end{abstract}

\section{INTRODUCTION}

A PBG cavity is formed using a 2D lattice of metal rods with a defect (missing rod) in the center. The lattice is placed between two flat, circular endplates. A sequence of PBG cavities with beam holes in the endplates can be employed as an accelerator structure [1] or possibly as an output structure of a high power klystron. This design takes advantage of the PBG structure to localize the defect mode while higher-order, higher-frequency modes are not localized but, instead, are spread throughout the volume of the cavity. Therefore, wakefields in the linear accelerator as well as spurious oscillations in the klystron, which are formed by higher-order modes, can be sufficiently suppressed.

\section{PBG CAVITY DESIGN AND COLD TEST}

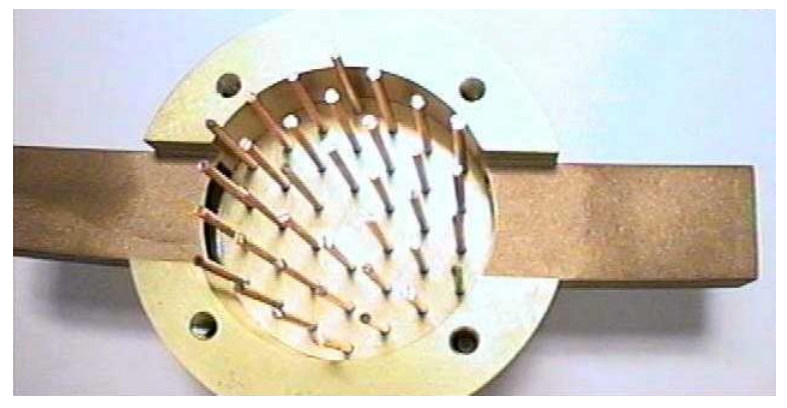

Fig. $1.17 \mathrm{GHz}$ photonic bandgap cavity built for cold test.

We report the results from a $17 \mathrm{GHz}$ PBG cavity using a triangular lattice of rods. Figure 1 shows the photograph of the cavity with the top cover removed. The cavity was built for cold test study. Three rows of rods are utilized to localize the defect mode. The defect mode is a quasi$\mathrm{TM}_{010}$ mode, analogous to the fundamental mode of a pillbox cavity. The electric field is parallel to the rods.

The SUPERFISH code [2] was employed to design the PBG cavity. The resonant frequency, Ohmic Q-factor and
Table 1 . PBG cavity and pillbox cavity parameters.

\begin{tabular}{|l|l|l|}
\hline & $\begin{array}{l}\text { PBG } \\
\text { Cavity }\end{array}$ & $\begin{array}{l}\text { Pillbox } \\
\text { Cavity }\end{array}$ \\
\hline Eigenfrequency $(\mathrm{GHz})$ & 17.3 & 17.3 \\
\hline Lattice spacing $b(\mathrm{~cm})$ & 0.64 & \\
\hline Rod radius $a(\mathrm{~cm})$ & 0.079 & \\
\hline Outer radius $(\mathrm{cm})$ & 2.15 & 0.657 \\
\hline Ohmic Q-factor $Q_{\text {ohm }}$ & 5200 & 7100 \\
\hline Shunt impedance $r_{c y}(\mathrm{M} \Omega / \mathrm{cm})$ & 2.1 & 2.9 \\
\hline $\begin{array}{l}\text { Maximum magnetic field ratio } \\
\eta=H_{\text {max }} Z_{\delta} E_{\text {max }}\end{array}$ & 1.3 & 0.94 \\
\hline $\begin{array}{l}\text { Frequency shift due to } \\
\text { coupling (MHz) }\end{array}$ & 30 & 300 \\
\hline $\begin{array}{l}\text { Pulse heating } \Delta \mathrm{T}(\text { deg. C) for } \\
\text { 1MW, } 100 \mathrm{~ns} \text { pulse }\end{array}$ & 50 & 25 \\
\hline $\begin{array}{l}\text { Max. longitudinal wake } \\
\text { potential (V/pC per cell) }\end{array}$ & 8.6 & 16. \\
\hline $\begin{array}{l}\text { Max. transverse dipole mode } \\
\text { wake potential (V/pC per cell) }\end{array}$ & 2.7 & 56. \\
\hline
\end{tabular}

shunt impedance of the PBG cavity were calculated. The dimensions and simulation results for both PBG and pillbox cavities are shown in Table 1 .

A vector network analyzer was employed to measure the PBG cavity. During the measurements we focused on the problem of coupling into the PBG cavity. RF power is coupled into the PBG cavity from the WR62 rectangular waveguide (Fig. 1). We measured the loaded and Ohmic Q-factor of the PBG cavity using the scattering matrix element $S_{11}$ frequency dependence. We removed some rods to control the coupling into the cavity. The PBG cavity is undercoupled as designed. Further coupling correction was accomplished when some rods were partially withdrawn. Such a coupling correction is a unique feature of PBG cavities and cannot be done with a conventional pillbox cavity. The critical coupling is observed experimentally in cold test

We run the High Frequency Structure Simulator (HFSS) [3], a 3D electromagnetic code, to model the experiment. Good agreement between the simulations and measurements was found. The HFSS simulations indicate that the coupling from the waveguide results in a very small frequency shift in the PBG cavity compared to that in the pillbox cavity (Table 1). The field is mainly confined by the first row of rods in the PBG cavity, which are not disturbed for achieving coupling into the cavity. 
Hence coupling does not cause eigenmode field variations or frequency shifts.

The cold test and simulation results are presented in more detail in [4]. Also, the properties of the 2D metal rod lattices, particularly, the global band gaps are reported by Smirnova et al. at this conference [5].

\section{PULSED HEATING}

The temperature rise at the rod surface can be estimated using the equation [6]:

$$
\Delta T=\frac{R_{s}}{2 K} \sqrt{D \tau} H_{\max }^{2}
$$

where $K=3.95 \mathrm{~W} /(\mathrm{cm} \mathrm{deg} . \mathrm{C})$ is the thermal conductivity of copper, $D=1.15 \mathrm{~cm}^{2} / \mathrm{s}$ is the thermal diffusion coefficient of copper, $R_{s}=0.034 \Omega$ is the surface resistance of copper at $17 \mathrm{GHz}$, and $\tau$ is the pulse length. The maximum magnetic field $H_{\max }$ is determined by the dissipated $\mathrm{rf}$ power $P$, the shunt impedance $r_{c y}$, the magnetic field enhancement ratio $\eta$ (Table 1), and the axial length $L$ :

$$
H_{\max }=\frac{\eta}{Z_{0}} \sqrt{\frac{r_{c y} P}{L}}
$$

where $Z_{0}=377 \Omega$. For the $\Delta T$ shown in Table 1 , it is assumed that the rf power is $1 \mathrm{MW}$, which corresponds to the unloaded gradient of about $80 \mathrm{MV} / \mathrm{m}$, the pulse length is $100 \mathrm{~ns}$, and $L=0.79 \mathrm{~cm}$ for the experimental cavity.

\section{WAKEFIELD POTENTIAL}

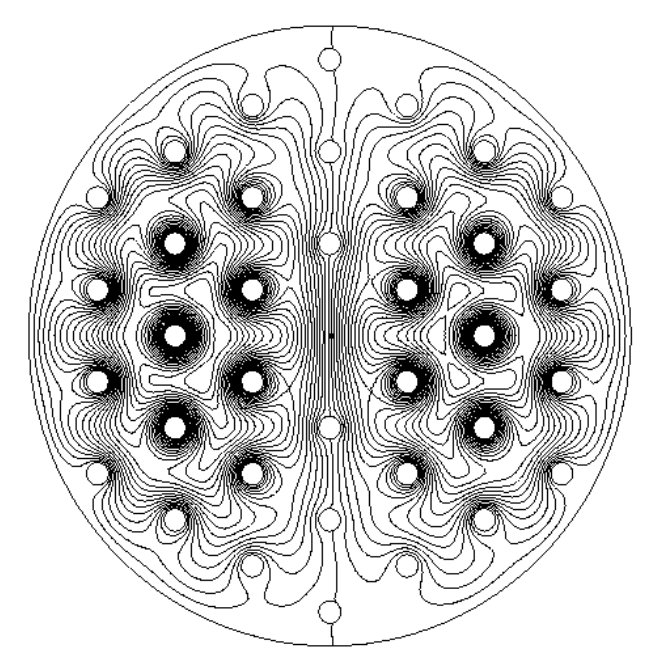

Fig. 2. SUPERFISH simulation of the $\mathrm{TM}_{110}$ mode in the PBG cavity. Contours of axial electric field are plotted.

The wakefields in the PBG structure have been estimated using the $2 \mathrm{D}$ code SUPERFISH. The $\mathrm{TM}_{\mathrm{on} 0}$ axially symmetrical (monopole) modes and $\mathrm{TM}_{1 \mathrm{n} 0}$ dipole modes are calculated in the PBG and pillbox cavities. Using the distributions of the longitudinal component of the electric field $E_{z}$, we calculate the longitudinal loss factors $k_{n}$ and transverse dipole-mode kick factors $k_{n}^{(1)}$ corresponding to different modes [7,8]. Furthermore, we calculate the longitudinal $\left(W_{z}\right)$ and transverse $\left(W_{\perp}\right)$ wake potentials formed by the driving charge at the test charge position, at some distance $s$ behind the driving charge. A short bunch approximation is used to calculate the wake potentials. Each of the longitudinal and transverse wake potentials is a result of summation of the loss (or kick) factors from all the modes. Here are the expressions for the wake potentials $[7,8]$ :

$$
\begin{aligned}
& W_{z}=2 \sum_{n} k_{n} \cos \left(\omega_{n} s / c\right) \\
& k_{n}=\frac{E_{z n}^{2}(0)}{4 u_{n}} T_{n}^{2} L \\
& W_{\perp}=2 \sum_{n} \frac{k_{n}^{(1)} c}{\omega_{n} a_{i r}} \sin \left(\omega_{n} s / c\right) \\
& k_{n}^{(1)}=\frac{E_{z n}^{2}\left(r=a_{i r}\right)}{4 u_{n}} L
\end{aligned}
$$

The $\mathrm{TM}_{0 \mathrm{n}}$ modes, with the frequencies $\omega_{n}$ and the electric fields at the axis $E_{z}(0)$, contribute to the longitudinal wake potential (3). In Eqs. (4) and (6), $u_{n}$ denotes the $n$-th mode energy per unit length (the SUPERFISH output parameter). The longitudinal wakefield potential in the disk-loaded accelerating structure is modeled using the 2D simulation results. The loss factor (4) includes the length of the cell $L$, which is determined as $L=0.583 \mathrm{~cm}$ for the $17 \mathrm{GHz}$ disk-loaded structure. Also, Eq.(4) includes the transit angle factors $T_{n}$ of the $\mathrm{TM}_{\mathrm{on} 0}$ modes in the cell. We assume that the modes have the sinusoidal axial distribution. Therefore, the expression for the transit angle factors is the following:

$$
T_{n}=\left|\frac{2 \lambda_{n}^{2} \cos \left(\pi L / \lambda_{n}\right)}{\pi\left(4 L^{2}-\lambda_{n}^{2}\right)}\right|
$$

The dipole $\mathrm{TM}_{\mathrm{In} 0}-$ like modes contribute to the transverse wake potential (5). In Eq. (6), the electric field is taken at the inner radius of the iris $a_{i r}$.

We simulated the wakefield potentials in both PBG and pillbox structures designed at $17 \mathrm{GHz}$. The dimensions of the irises were scaled from the SLAC 2.856 $\mathrm{GHz}$ design [7], therefore, $a_{i r}=0.19 \mathrm{~cm}$, and the disk thickness is $0.096 \mathrm{~cm}$. We also simulated the wakefields in the SLAC $2.856 \mathrm{GHz}$ structure and found the results consistent to the SLAC simulations [8]. The simulation of the wake potentials of the $17 \mathrm{GHz}$ pillbox structure confirmed that the wake potential per cell $\left(W_{z} L\right.$ and $\left.W_{\perp} L\right)$ scales as the operating frequency, i.e. larger by a factor of 6 than that in the $2.856 \mathrm{GHz}$ structure. 
We found that the loss factor of the fundamental $\mathrm{TM}_{010}$ mode in the PBG structure is of the same order as that in the $17 \mathrm{GHz}$ pillbox structure. However, the loss factors for the higher-order $\mathrm{TM}_{0 \mathrm{n} 0}$ modes are smaller.

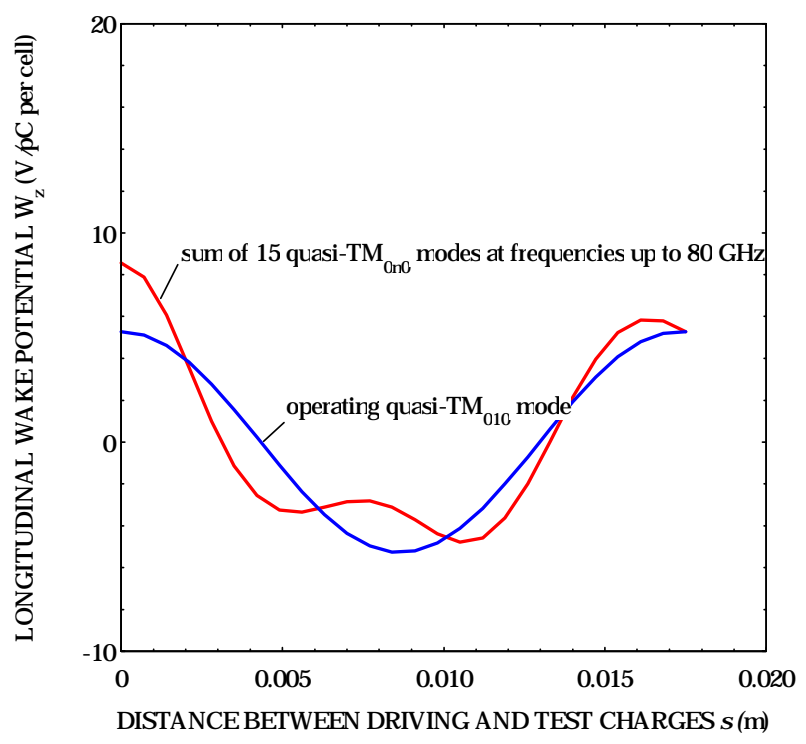

Fig. 3. Longitudinal wake potential (per cell) in the PBG accelerating structure.

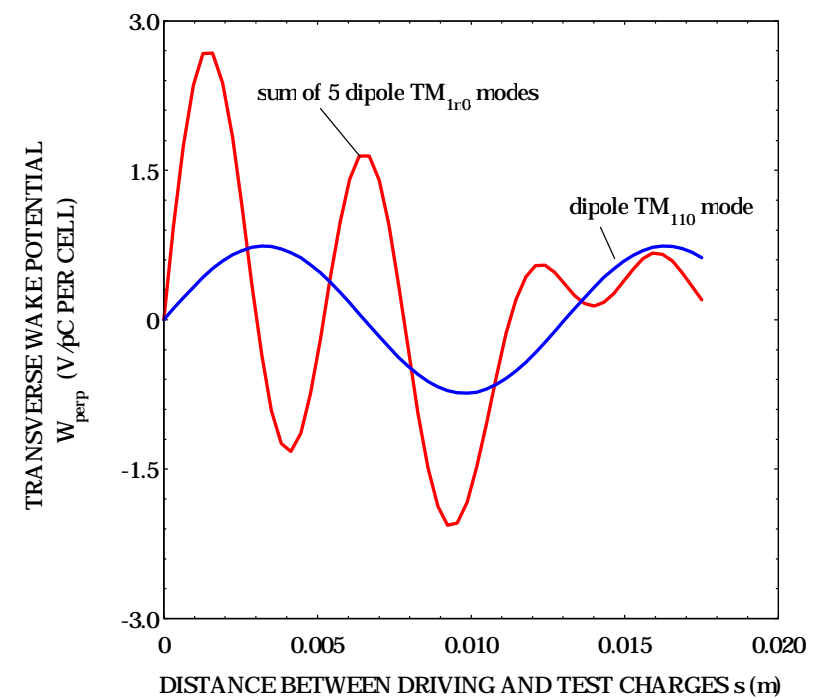

Fig. 4. Transverse wake potential (per cell) in the PBG accelerating structure.

As a result, the maximum longitudinal wake potential is smaller by a factor of 2 in the PBG structure (Table 1).

The dipole quasi- $\mathrm{TM}_{1 \mathrm{n} 0}$ modes in the $2 \mathrm{D}$ PBG structure are determined to calculate the transverse wake potential. The quasi-TM $\mathrm{TM}_{110}$ mode of the PBG structure found in the SUPERFISH simulations is displayed in Fig.2. The dipole mode penetrates through the metal array and occupies the entire area of the PBG cavity. As a result transverse wake potential in the PBG cavity is smaller than that in the pillbox cavity by a factor of 20 (Table 1). The calculated longitudinal wake potential as a function of the distance between the driving and test charges is presented in Fig. 3, and the transverse wake potential is plotted in Fig. 4. In these calculations, we assume that the iris radius in the PBG structure is the same as that in the $17 \mathrm{GHz}$ pillbox structure.

\section{CONCLUSIONS}

The PBG cavity was studied numerically and in cold test and demonstrated important features that make the cavity useful for applications in linear accelerators. The waveguide coupling into the PBG cavity can be corrected during the cold test with no frequency shift. Preliminary 2D calculations of the wakefield potentials have demonstrated high order mode wakefield suppression in the PBG cavity. More rigorous calculations will be done using the 3D code HFSS for the PBG accelerating structure including the beam holes.

This work was supported by the US Dept. of Energy, HEP Contract No. DE-FG02-91ER40648.

\section{REFERENCES}

[1] D.R.Smith, N.Kroll, and S.Schultz, Advanced Accelerator Concepts, ed. P.Schoessow, AIP Conf. Proc. No. 335 (1995), p. 761.

[2] J.H.Billen and L.M.Young, LANL Report No. LAUR-96-1834, 1996.

[3] HFSS Manual, ANSOFT Corp., 1999.

[4] M.A.Shapiro et al., Phys. Rev. Special Topics Accelerators and Beams, Vol. 4, 042001 (2001).

[5] E.I.Smirnova et al., PAC'2001.

[6] P.B.Wilson, "Scaling Linear Colliders to $5 \mathrm{TeV}$ and Above," SLAC-PUB-7449, April 1997, 12 p.

[7] P.B.Wilson, Applications of High-Power Microwaves, ed.: A.V.Gaponov-Grekhov and V.L.Granatstein, 1994, pp. 229-317.

[8] P.B.Wilson, "Introduction to Wakefields and Wake Potentials," SLAC-PUB-4547, January 1989, 39 p. 